\title{
A PREY-PREDATOR SYSTEM WITH HERD BEHAVIOUR OF PREY IN A RAPIDLY FLUCTUATING ENVIRONMENT
}

\author{
GURUPRASAD SAMANTA, ASHOK MONDAL, DEBGOPAL SAHOO, AND PRALAY DOLAI
}

\begin{abstract}
A statistical theory of non-equilibrium fluctuation in damped Volterra-Lotka prey-predator system where prey population lives in herd in a rapidly fluctuating random environment has been presented. The corresponding results have also been obtained in absence of herd behaviour. The method is based on the technique of perturbation approximation of non-linear coupled stochastic differential equations. The characteristic of group-living of prey population has been emphasized using square root of prey density in the functional response. Numerical results have also been obtained by varying some of the vital system parameters.
\end{abstract}

\section{INTRODUCTION}

The classical Volterra-Lotka equations are generally used to describe the time-evolution of interacting prey-predator system. The introduction of intraspecific competition among the prey, resulting from the limited resources, makes the Volterra-Lotka model rough and the system is known as the damped Volterra-Lotka system. In natural ecosystems, it has been observed that most of the prey populations live forming groups, and all members of a group do not interact at a time (Bera et al. 2015, 2016a, 2016b). Major predators of zebra, buffalo, kongoni, toki and Thomsons gazelle are hyena, wild dog, lion, leopard and cheetahs and so to defend against predators they form groups. The underlying reasons behind group formation more likely depend upon self-defence, group-defence, group alertness within a group and speed, to avoid being killed by a predator (Khan et al. 2004). Models of group formation are analysed to study environmental and social forces, and individual decision rules that lead to formation of swarms, flocks, schools, herds, and other groups (DeAngelis and Mooij 2005). It is pointed out by Fryxell et al. (2007) that group formation profoundly reduce food intake rates below the expected levels. As a consequence, suitable form of functional response was searched by researchers to describe the social behaviour of such populations. Freedman and Wolkowitz (1986) analysed the characteristics of group defence in this regard. Now, when a population lives forming groups, then all members of a group do not interact at a time and some of the reasons for this herd behaviour are for searching food resources, defending the predators. To explore the consequence of spatial group formation of fixed shape by predators, Cosner et al. (1999) introduced the idea that the square root of the predator variable is to be used in the function describing the encounter rate in two-dimensional systems. Unfortunately, such an idea has not been incorporated by the researchers for about a decade. The most significant works of Ajraldi et al. (2011) and Braza (2012) gave such modelling a new dimension. The central ideas are as follows: Let $x$ be the density of a population that gathers in herds, and suppose that herd occupies an area $A$. The number of individuals staying at outermost positions in the herd is proportional to

Received by the editors 4 August 2019; revised 12 November 2019; accepted 20 November 2019, published online 6 December, 2019.

2000 Mathematics Subject Classification. 92D25; 37H10.

Key words and phrases. Herd, random environment, coloured noise, white noise, stability. 
the length of the perimeter of the patch where the herd is located. Clearly, its length is proportional to $\sqrt{A}$. Since $x$ is distributed over a two-dimensional space, $\sqrt{x}$ would therefore count the individuals at the edge of the patch. As a result, the encounter rate $e(x, y)=\beta x y$ should change its form to $e(x, y)=\beta \sqrt{x} y$ in two dimension.

Rapidly fluctuating environmental variations usually cause random variations in system parameters, in particular, in the natural growth coefficient of the prey and in the natural mortality of the predator. Since these are the main parameters subject to coupling of a prey-predator pair with its environment (Dimentberg 1988). Bera et al. (2016a) considered a prey-predator model, where the 'functional response' is of the form Holling type-II, but the prey density is replaced by its square root. They studied the stochastic version of the model, which takes into account the effect of fluctuating environment characterized by Gussion white noises. A prey-predator model was proposed in the work of Maiti et al.(2016), where both the prey and the predator show herd behaviour. The effect of fluctuating environment was analyzed by them incorporating Gaussian white noises. They concluded that, to keep ecological balance in a fluctuating environment, the system has to maintain some restrictions. In the present article we have developed a general stochastic analysis of the behaviour of the damped Volterra-Lotka prey-predator system with herd behaviour in prey population in a rapidly fluctuating random environment. The method is based on the technique of perturbation approximation of nonlinear coupled stochastic differential equations. Numerical results have also been obtained by varying some of the vital system parameters. We have derived the corresponding results in absence of herd behaviour.

\section{Damped Volterra-Lotka System With herd Behaviour of PREy: Basic STOCHastic DIFFERENTIAL EQUATIONS}

For damped Volterra-Lotka system with herd behaviour of prey, the prey population represented by its biomass $x(t)$ and that of predator population represented by $y(t)$ satisfy the following deterministic equation:

$$
\frac{d}{d t} X(t)=F(X(t))
$$

where

$$
X(t)=\left[\begin{array}{l}
x(t) \\
y(t)
\end{array}\right], \quad F=\left[\begin{array}{c}
x(t)(\alpha-k x(t))-\beta \sqrt{x(t)} y(t) \\
y(t)(-m+\eta \beta \sqrt{x(t)})
\end{array}\right], \text { and } \alpha, m, k, \eta, \beta>0 .
$$

It is assumed that fluctuations in the environment will manifest themselves mainly as fluctuations in the natural growth coefficient of the prey $(\alpha)$ and in the natural mortality of the predator $(-m)$, because these are the main parameters subject to coupling of a prey-predator pair with its environment (Samanta and Maiti 2003). In a random environment, the parameters $\alpha$ and $-m$ are replaced by $\alpha+\theta_{1}(t)$ and $-m+\theta_{2}(t)$ respectively, where $\theta_{1}(t)$ and $\theta_{2}(t)$ are random fluctuating terms. We assume that these fluctuations are rapid and we express this fact by writing $\theta(\tau) \equiv\left(\theta_{1}(\tau), \theta_{2}(\tau)\right)$ where $\tau=t / \epsilon$ and $0<\epsilon<<1$ is a small, non-random parameter.

In a rapidly fluctuating random environment, the stochastic modification of (1) is as follows:

$$
\frac{d}{d t} X(\epsilon, t)=F(X(\epsilon, t), \theta(t / \epsilon))
$$


where

$$
X(\epsilon, t)=\left[\begin{array}{l}
x(\epsilon t) \\
y(\epsilon, t)
\end{array}\right], \quad F(X(\epsilon, t), \theta(t / \epsilon))=\left[\begin{array}{c}
x(\epsilon, t)\left(\alpha+\theta_{1}(t / \epsilon)-k x(\epsilon, t)\right)-\beta \sqrt{x(\epsilon, t)} y(\epsilon, t) \\
y(\epsilon, t)\left(-m+\theta_{2}(t / \epsilon)+\eta \beta \sqrt{x(\epsilon, t)}\right)
\end{array}\right],
$$

$x(\epsilon, t), y(\epsilon, t)$ represent the prey and predator population respectively and $\alpha, m, k, \eta, \beta>0 ; 0<\epsilon<<1$.

The meaning of this is as follows: as the natural time $t$ changes by a typical amount $\delta t, \theta(t / \epsilon)$ fluctuates considerably, since it experiences an elapsed time $\delta \tau=\frac{\delta t}{\epsilon}$ which is large when $\epsilon$ is small. We assume that the perturbed terms $\theta_{1}(\tau), \theta_{2}(\tau) ; \tau=t / \epsilon$, are coloured noises or Ornstein-Uhlenbeck processes. The mathematical expectations and correlation functions of these processes are given by

$$
\begin{aligned}
& \left\langle\theta_{i}(\tau)\right\rangle=0,\left\langle\theta_{i}\left(\tau_{1}\right) \theta_{i}\left(\tau_{2}\right)\right\rangle=\frac{\sigma_{i}^{2}}{2 \gamma_{i}} \exp \left(-\gamma_{i}\left|\tau_{1}-\tau_{2}\right|\right), \\
& \left\langle\theta_{1}\left(\tau_{1}\right) \theta_{2}\left(\tau_{2}\right)\right\rangle=\sigma \exp \left(-\left|\tau_{1}-\tau_{2}\right|\right)\left(1+\left|\tau_{1}-\tau_{2}\right|\right),\left(\gamma_{i}>0, i=1,2\right),
\end{aligned}
$$

where $\langle\cdot\rangle$ represents the average over the ensemble of the stochastic process.

This is motivated by the fact that

$$
\lim _{\left|\tau_{1}-\tau_{2}\right| \rightarrow \infty}\left\langle\theta_{1}\left(\tau_{1}\right) \theta_{2}\left(\tau_{2}\right)\right\rangle=0 \Rightarrow \theta_{1}\left(\tau_{1}\right), \theta_{2}\left(\tau_{2}\right) \text { tend to independent random processes. }
$$

It is also noted that as $\sigma_{i}, \gamma_{i} \rightarrow \infty$ keeping $\frac{\sigma_{i}^{2}}{\gamma_{i}^{2}}=$ constant $=D_{i}^{2}$ (say), then $\theta_{i}(\tau) \rightarrow D_{i} \eta_{i}(\tau)$ where $\eta_{i}(\tau)$ are standard white noises, i.e., $\left\langle\eta_{i}(\tau)\right\rangle=0,\left\langle\eta_{i}\left(\tau_{1}\right) \eta_{i}\left(\tau_{2}\right)\right\rangle=\delta\left(\tau_{1}-\tau_{2}\right)$.

\section{Perturbation approximation and non-equilibrium fludtuation}

We shall now use a two term perturbation approximation to $X(\epsilon, t)$ (White 1977):

$$
X(\epsilon, t) \sim X^{0}(t)+\sqrt{\epsilon} Y^{0}(t) .
$$

The first approximation

satisfies

$$
X^{0}(t)=\left[\begin{array}{l}
x^{0}(t) \\
y^{0}(t)
\end{array}\right]
$$

where

$$
\frac{d}{d t} X^{0}(t)=\bar{F}\left(X^{0}(t)\right)
$$

$$
\begin{gathered}
\bar{F}\left(X^{0}(t)\right)=\lim _{T \rightarrow \infty} \frac{1}{T} \int_{0}^{T}\left\langle F\left(X^{0}(t), \theta(\tau)\right)\right\rangle d \tau \\
=\left[\begin{array}{c}
x^{0}(t)\left(\alpha-k x^{0}(t)\right)-\beta \sqrt{x^{0}(t)} y^{0}(t) \\
y^{0}(t)\left(-m+\eta \beta \sqrt{x^{0}(t)}\right)
\end{array}\right] .
\end{gathered}
$$

These are just the equations of the damped Volterra-Lotka system with herd behaviour of prey in a fixed environment. This system has a unique non-trivial equilibrium (both components of which are non-zero) at the point:

$$
\bar{X}=\left[\begin{array}{c}
x^{\star} \\
y^{\star}
\end{array}\right], \text { where } x^{\star}=\frac{m^{2}}{\eta^{2} \beta^{2}}, y^{\star}=\frac{\alpha \eta^{2} \beta^{2}-k m^{2}}{\eta^{3} \beta^{4}} .
$$

It is immediately apparent that in the absence of predators the limit value of prey population will be $x^{\prime}=\frac{\alpha}{k}$. The realization of the obvious condition: 


$$
x^{\star}<x^{\prime} \Rightarrow \alpha \eta^{2} \beta^{2}-k m^{2}>0
$$

which makes $y^{\star}$ positive, and hence this equilibrium exists.

We assume that the system is at the initial time $t=0$ at $\bar{X}$, therefore we have $X^{0}(t)=\bar{X}$. Here

$$
Y^{0}(t)=\left[\begin{array}{c}
Y_{1}^{0}(t) \\
Y_{2}^{0}(t)
\end{array}\right]
$$

is a Gaussian random process which satisfies the linear equation

$$
\frac{d}{d t} Y^{0}(t)=C Y^{0}(t)+W(t)
$$

where

$$
Y^{0}(0)=0, C=\frac{\partial \bar{F}}{\partial X}(\bar{X})=\left[\begin{array}{cc}
-a & -\frac{m}{\eta} \\
b & 0
\end{array}\right]
$$

where

and

$$
a=\frac{2 m\left(2 k m^{2}-\alpha \eta^{2} \beta^{2}\right)+\left(\alpha \eta^{2} \beta^{2}-k m^{2}\right)}{2 m \eta^{2} \beta^{2}}, b=\frac{\alpha \eta^{2} \beta^{2}-k m^{2}}{2 m \eta \beta^{2}}
$$

$$
\begin{gathered}
\langle W(t)\rangle=0,\left\langle W(t) W^{T}\left(t^{\prime}\right)\right\rangle=A \delta\left(t-t^{\prime}\right), \\
A=\lim _{T \rightarrow \infty} \frac{1}{T} \int_{0}^{T} \int_{0}^{T} \begin{array}{c}
\left\langle\left[F\left(\bar{X}, \theta\left(\tau_{1}\right)\right)-\left\langle F\left(\bar{X}, \theta\left(\tau_{1}\right)\right)\right\rangle\right]\right. \\
\left.\left[F\left(\bar{X}, \theta\left(\tau_{2}\right)\right)-\left\langle F\left(\bar{X}, \theta\left(\tau_{2}\right)\right)\right\rangle\right]^{T}\right\rangle d \tau_{1} d \tau_{2}
\end{array} \\
=\left[\begin{array}{ll}
A_{11} & A_{12} \\
A_{21} & A_{22}
\end{array}\right], \\
A_{11}=\frac{m^{4} \sigma_{1}^{2}}{\eta^{4} \beta^{4} \gamma_{1}^{2}}, A_{12}=A_{21}=\frac{4 m^{2}\left(\alpha \eta^{2} \beta^{2}-k m^{2}\right) \sigma}{\eta^{5} \beta^{6}}, A_{22}=\frac{\left(\alpha \eta^{2} \beta^{2}-k m^{2}\right)^{2} \sigma_{2}^{2}}{\eta^{6} \beta^{8} \gamma_{2}^{2}} .
\end{gathered}
$$

Now the solution of (8) is given by

$$
Y^{0}(t)=Y(t) \int_{0}^{t} Y^{-1}(s) W(s) d s,
$$

where $Y(t)$ satisfies the linear equation:

$$
\frac{d}{d t} Y(t)=C Y(t), Y(0)=I
$$

Therefore,

$$
\left\langle Y^{0}(t)\right\rangle=0, \text { since }\langle W(s)\rangle=0 .
$$

The solution of (12) is given by

$$
Y(t)=\left[\begin{array}{cc}
\frac{1}{\sqrt{\Delta}}\left(\lambda_{1} e^{\lambda_{1} t}-\lambda_{2} e^{\lambda_{2} t}\right) & -\frac{m}{\eta \sqrt{\Delta}}\left(e^{\lambda_{1} t}-e^{\lambda_{2} t}\right) \\
\frac{b}{\sqrt{\Delta}}\left(e^{\lambda_{1} t}-e^{\lambda_{2} t}\right) & -\frac{1}{\sqrt{\Delta}}\left(\lambda_{2} e^{\lambda_{1} t}-\lambda_{1} e^{\lambda_{2} t}\right)
\end{array}\right],
$$

where ( $a$ and $b$ are given by (10)):

$$
\Delta=a^{2}-\frac{4 m}{\eta} b, \lambda_{1}=\frac{-a+\sqrt{\Delta}}{2}, \lambda_{2}=\frac{-a-\sqrt{\Delta}}{2} .
$$


Let us assume that

$$
2 k m^{2}-\alpha \eta^{2} \beta^{2}>0 \Rightarrow k m^{2}<\alpha \eta^{2} \beta^{2}<2 k m^{2} \Rightarrow a>0 .
$$

Hence the system (1) is locally asymptotically stable at the unique non-trivial equilibrium point given by $(6)$.

Now the expression of the strength of the fluctuation $D(t)$, the covariance at one instant of time, is given by

$$
\begin{aligned}
D(t)=\left\langle Y^{0}(t) Y^{0^{T}}(t)\right\rangle & =Y(t)\left[\int_{0}^{t} Y^{-1}(s) A Y^{-1^{T}}(s) d s\right] Y^{T}(t) \\
& =\left[\begin{array}{ll}
D_{11}(t) & D_{12}(t) \\
D_{21}(t) & D_{22}(t)
\end{array}\right]
\end{aligned}
$$

where

$$
\begin{aligned}
& D_{11}(t)=\frac{e^{2 \lambda_{1} t}}{2 \Delta b} \Phi\left(\lambda_{1}, \lambda_{2}\right)+\frac{e^{2 \lambda_{2} t}}{2 \Delta b} \Phi\left(\lambda_{2}, \lambda_{1}\right)+\frac{2 m}{\Delta \eta} e^{-a t} \Psi+\frac{1}{2 a b}\left\{A_{11} b+A_{22} \frac{m}{\eta}\right\}, \\
& D_{12}(t)=D_{21}(t)=\frac{e^{2 \lambda_{1} t}}{2 \Delta \lambda_{1}} \Phi\left(\lambda_{1}, \lambda_{2}\right)+\frac{e^{2 \lambda_{2} t}}{2 \Delta \lambda_{2}} \Phi\left(\lambda_{2}, \lambda_{1}\right)-\frac{e^{-a t}}{2 \Delta b} \Theta-\frac{A_{22}}{2 b}, \\
& D_{22}(t)=\frac{e^{2 \lambda_{1} t}}{2 \Delta \lambda_{1}} Z\left(\lambda_{2}\right)+\frac{e^{2 \lambda_{2} t}}{2 \Delta \lambda_{2}} Z\left(\lambda_{1}\right)+\frac{2 b}{\Delta} e^{-a t} \Psi+M, \\
& \Phi(x, y)=A_{11} b x-2 A_{12} x y+A_{22} \frac{m}{\eta} y, \Psi=\frac{1}{a}\left\{A_{11} b+A_{22} \frac{m}{\eta}\right\}+A_{12}, \\
& \Theta=2 A_{11} b^{2}+2 A_{12} a b+A_{22}\left\{a^{2}+2 \frac{m}{\eta} b\right\} \\
& Z(x)=A_{11} b^{2}-2 A_{12} b x+A_{22} x^{2}, \\
& M=\frac{A_{11} b \eta}{2 a m}+\frac{A_{22} \eta}{2 a m b}\left\{a^{2}+\frac{m}{\eta} b\right\}+\frac{A_{12}}{m} \eta .
\end{aligned}
$$

Therefore, $D(t)$ converges exponentially to the limiting variance

$$
D(\infty)=\left[\begin{array}{ll}
D_{11}(\infty) & D_{12}(\infty) \\
D_{21}(\infty) & D_{22}(\infty)
\end{array}\right]
$$

where 


$$
\begin{aligned}
& D_{11}(\infty)=\frac{m^{3}}{\left\{2 m\left(2 k m^{2}-\alpha \eta^{2} \beta^{2}\right)+\left(\alpha \eta^{2} \beta^{2}-k m^{2}\right)\right\} \eta^{2} \beta^{2}}\left[m^{2} \frac{\sigma_{1}^{2}}{\gamma_{1}^{2}}\right. \\
& \left.+\frac{2\left(\alpha \eta^{2} \beta^{2}-k m^{2}\right)}{\eta^{2} \beta^{2}} \frac{\sigma_{2}^{2}}{\gamma_{2}^{2}}\right], \\
& D_{12}(\infty)=D_{21}(\infty)=-\frac{m\left(\alpha \eta^{2} \beta^{2}-k m^{2}\right)}{\eta^{5} \beta^{6}} \frac{\sigma_{2}^{2}}{\gamma_{2}^{2}}, \\
& D_{22}(\infty)=\frac{m^{3}\left(\alpha \eta^{2} \beta^{2}-k m^{2}\right)}{2\left\{2 m\left(2 k m^{2}-\alpha \eta^{2} \beta^{2}\right)+\left(\alpha \eta^{2} \beta^{2}-k m^{2}\right)\right\} \eta^{2} \beta^{4}} \frac{\sigma_{1}^{2}}{\gamma_{1}^{2}}+ \\
& {\left[\frac{\left(\alpha \eta^{2} \beta^{2}-k m^{2}\right)\left\{2 m\left(2 k m^{2}-\alpha \eta^{2} \beta^{2}\right)+\left(\alpha \eta^{2} \beta^{2}-k m^{2}\right)\right\}}{2 m \eta^{6} \beta^{8}}+\right.} \\
& \left.\frac{m\left(\alpha \eta^{2} \beta^{2}-k m^{2}\right)^{2}}{\left\{2 m\left(2 k m^{2}-\alpha \eta^{2} \beta^{2}\right)+\left(\alpha \eta^{2} \beta^{2}-k m^{2}\right)\right\} \eta^{4} \beta^{6}}\right] \frac{\sigma_{2}^{2}}{\gamma_{2}^{2}} \\
& +\frac{4 m\left(\alpha \eta^{2} \beta^{2}-k m^{2}\right) \sigma}{\eta^{4} \beta^{6}} .
\end{aligned}
$$

This convergence is rapid. In the case of high-amplitude fluctuations $D_{11}(\infty)$ and $D_{22}(\infty)$ are large. This indicates that for high-amplitude fluctuations the system demonstrates statistical parametric shatter as a result of rapidly fluctuating environmental conditions, and the equilibrium, which is stable in absence of these fluctuations, becomes unstable. It is also evident from (15) that the prey population is more sensitive in rapidly fluctuating environmental conditions and the natural growth coefficient $(\alpha)$ of the prey play a significant role in instability. This is due to the herd behaviour of the prey population.

\section{Numerical Simulation FOR SyStem $(2)$}

\begin{tabular}{|c|c|c|c|c|c|c|c|c|c|}
\hline Parameter & $k$ & $\beta$ & $m$ & $\eta$ & $\sigma_{1}$ & $\gamma_{1}$ & $\sigma_{2}$ & $\gamma_{2}$ & $\sigma$ \\
\hline Value & 0.001 & 0.4 & 0.09 & 0.5 & 0.4 & 0.16 & 0.8 & 0.64 & 0.1 \\
\hline
\end{tabular}

Table-1: Parameter values used for numerical simulation of system (2) for Figure-1

If we change the parameter value of $\alpha$ continuously from 0.5 to 2.0 , limiting variance $D_{11}(\infty)$ decreases whereas $D_{22}(\infty)$ increases rapidly (see Figure-1), i.e., in a rapidly fluctuating environment, the interior equilibrium of system (2) which is stable in absence of these fluctuations, becomes unstable.

\begin{tabular}{|c|c|c|c|c|c|c|c|c|c|}
\hline Parameter & $\alpha$ & $k$ & $m$ & $\eta$ & $\sigma_{1}$ & $\gamma_{1}$ & $\sigma_{2}$ & $\gamma_{2}$ & $\sigma$ \\
\hline Value & 1.1 & 0.001 & 0.09 & 0.5 & 0.4 & 0.16 & 0.8 & 0.64 & 0.1 \\
\hline
\end{tabular}

Table-2: Parameter values used for numerical simulation of system (2) for Figure-2

Now taking $\alpha=1.1$ and varying the parameter value of $\beta$ from 0.2 to 2 , both the limiting variance $D_{11}(\infty)$ and $D_{22}(\infty)$ decreases and converges to a positive value which is very close to zero (see Figure2 ), i.e, the prey and the predator population coexist in stable equilibrium because the perturbation terms around the interior (coexistence) equilibrium given by (6) tend to zero. So, it can be concluded that for increasing consumption rate of predator the system develops an internal mechanism for coexistence steady state free from fluctuation though the environment is fluctuating rapidly. It is a very interesting result to maintain ecological balance, that is, to maintain the balance of nature. 

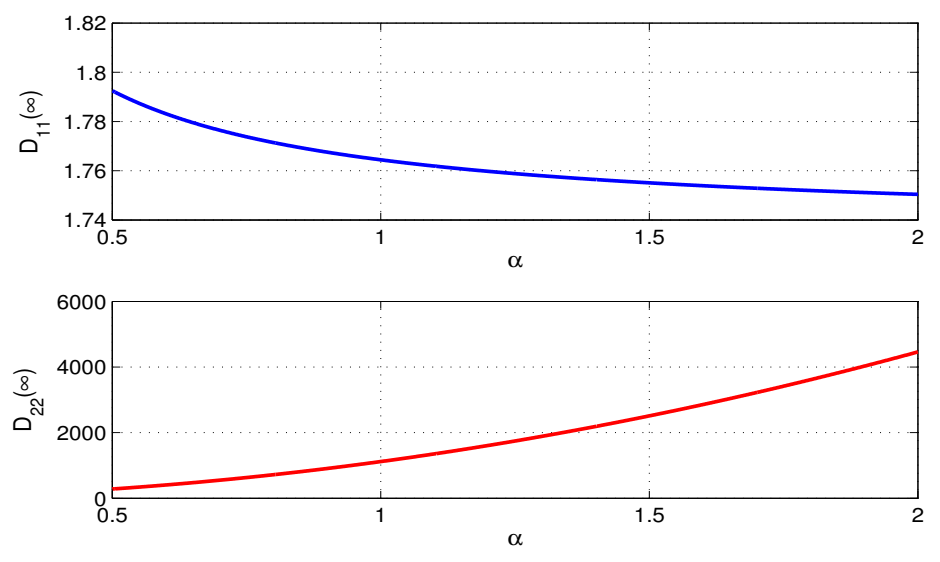

FIGURE 1. Limiting variance $D_{11}(\infty)$ and $D_{22}(\infty)$ with respect to the parameter value $\alpha$.
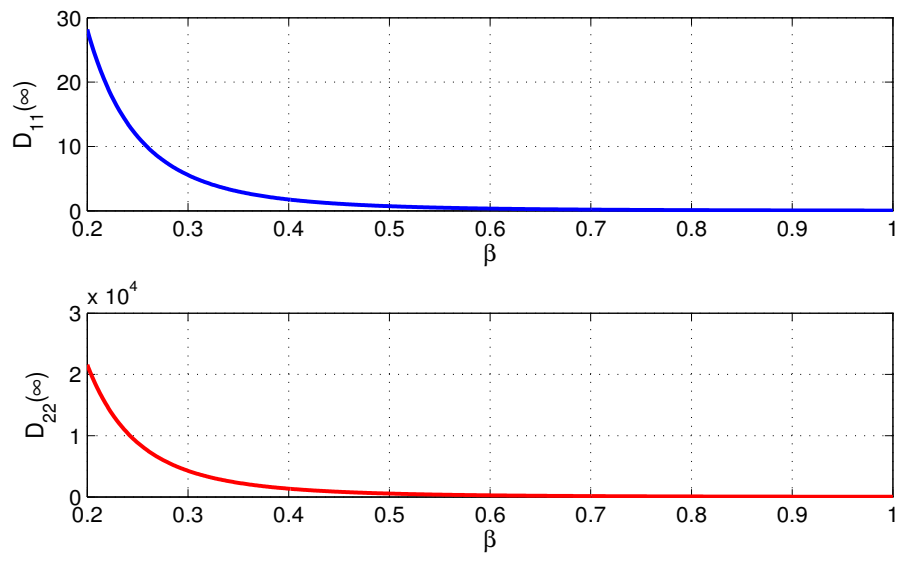

FiguRE 2. Limiting variance $D_{11}(\infty)$ and $D_{22}(\infty)$ with respect to the parameter value $\beta$.

\begin{tabular}{|c|c|c|c|c|c|c|c|c|c|}
\hline Parameter & $\alpha$ & $k$ & $\beta$ & $\eta$ & $\sigma_{1}$ & $\gamma_{1}$ & $\sigma_{2}$ & $\gamma_{2}$ & $\sigma$ \\
\hline Value & 1.1 & 0.001 & 0.4 & 0.5 & 0.4 & 0.16 & 0.8 & 0.64 & 0.1 \\
\hline
\end{tabular}

Table-3: Parameter values used for numerical simulation of system (2) for Figure-3

Now taking $\alpha=1.1, \beta=0.4$ and varying the predator death rate $m$ from 0.05 to 0.5 , both the limiting variance $D_{11}(\infty)$ and $D_{22}(\infty)$ increases rapidly near $m=0.5$ (see Figure-3), i.e, the system becomes unstable near $m=0.5$. Again around $m=0.2$ which is not too far from the rate $\eta \beta$, the limiting variances are small and so the predator and prey coexist in stable equilibrium which is free from fluctuation though the environment is fluctuating rapidly. This agrees with our theoretical findings.

\section{Damped Volterra-Lotka System without herd Behaviour: Basic Stochastic DIFFERENTIAL EQUATIONS}

In absence of herd behaviour of prey, system (1) takes the following form: 

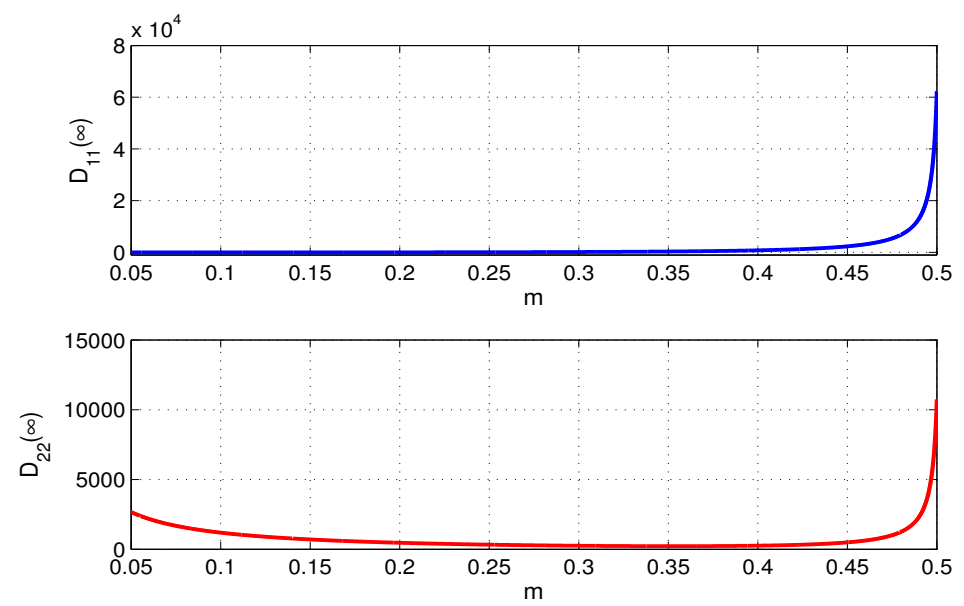

FIGURE 3. Limiting variance $D_{11}(\infty)$ and $D_{22}(\infty)$ with respect to the parameter value $m$.

$$
\frac{d}{d t} X(t)=F(X(t)),
$$

where

$$
X(t)=\left[\begin{array}{l}
x(t) \\
y(t)
\end{array}\right], \quad F=\left[\begin{array}{c}
x(t)(\alpha-k x(t)-\beta y(t)) \\
y(t)(-m+\eta \beta x(t))
\end{array}\right], \text { and } \alpha, m, k, \eta, \beta>0 .
$$

In a rapidly fluctuating random environment, system (16) is modified as follows:

$$
\frac{d}{d t} X(\epsilon, t)=F(X(\epsilon, t), \theta(t / \epsilon)),
$$

where

$$
X(\epsilon, t)=\left[\begin{array}{c}
x(\epsilon t) \\
y(\epsilon, t)
\end{array}\right], \quad F(X(\epsilon, t), \theta(t / \epsilon))=\left[\begin{array}{c}
x(\epsilon, t)\left(\alpha+\theta_{1}(t / \epsilon)-k x(\epsilon, t)-\beta y(\epsilon, t)\right) \\
y(\epsilon, t)\left(-m+\theta_{2}(t / \epsilon)+\eta \beta x(\epsilon, t)\right)
\end{array}\right],
$$

$x(\epsilon, t), y(\epsilon, t)$ represent the biomass of prey and predator population respectively and $\alpha, m, k, \eta, \beta>$ $0 ; 0<\epsilon<<1$. As in Section 2, the perturbed terms $\theta_{1}(\tau), \theta_{2}(\tau) ; \tau=t / \epsilon$, are coloured noises or Ornstein-Uhlenbeck processes characterized by (3).

Proceeding as in Section 3, the expression of the strength of the fluctuation $D(t)$, the covariance at one instant of time, is given by

$$
\begin{gathered}
D(t)=\left\langle Y^{0}(t) Y^{0^{T}}(t)\right\rangle=Y(t)\left[\int_{0}^{t} Y^{-1}(s) A Y^{-1^{T}}(s) d s\right] Y^{T}(t) \\
=\left[\begin{array}{ll}
D_{11}(t) & D_{12}(t) \\
D_{21}(t) & D_{22}(t)
\end{array}\right], \text { where } \\
D_{11}(t)=\frac{e^{2 \lambda_{1} t}}{2 \Delta b} \Phi\left(\lambda_{1}, \lambda_{2}\right)+\frac{e^{2 \lambda_{2} t}}{2 \Delta b} \Phi\left(\lambda_{2}, \lambda_{1}\right)+\frac{2 m}{\Delta \eta} e^{-a t} \Psi+\frac{1}{2 a b}\left\{A_{11} b+A_{22} \frac{m}{\eta}\right\},
\end{gathered}
$$




$$
\begin{aligned}
& D_{12}(t)=D_{21}(t)=\frac{e^{2 \lambda_{1} t}}{2 \Delta \lambda_{1}} \Phi\left(\lambda_{1}, \lambda_{2}\right)+\frac{e^{2 \lambda_{2} t}}{2 \Delta \lambda_{2}} \Phi\left(\lambda_{2}, \lambda_{1}\right)-\frac{e^{-a t}}{2 \Delta b} \Theta-\frac{A_{22}}{2 b}, \\
& D_{22}(t)=\frac{e^{2 \lambda_{1} t}}{2 \Delta \lambda_{1}} Z\left(\lambda_{2}\right)+\frac{e^{2 \lambda_{2} t}}{2 \Delta \lambda_{2}} Z\left(\lambda_{1}\right)+\frac{2 b}{\Delta} e^{-a t} \Psi+M \\
& \Phi(x, y)=A_{11} b x-2 A_{12} x y+A_{22} \frac{m}{\eta} y, \Psi=\frac{1}{a}\left\{A_{11} b+A_{22} \frac{m}{\eta}\right\}+A_{12}, \\
& \Theta=2 A_{11} b^{2}+2 A_{12} a b+A_{22}\left\{a^{2}+2 \frac{m}{\eta} b\right\} \\
& Z(x)=A_{11} b^{2}-2 A_{12} b x+A_{22} x^{2}, \\
& M=\frac{A_{11} b \eta}{2 a m}+\frac{A_{22} \eta}{2 a m b}\left\{a^{2}+\frac{m}{\eta} b\right\}+\frac{A_{12}}{m} \eta
\end{aligned}
$$

where

$$
A_{11}=\frac{m^{2} \sigma_{1}^{2}}{\eta^{2} \beta^{2} \gamma_{1}^{2}}, A_{12}=A_{21}=\frac{4 m(\eta \alpha \beta-k m) \sigma}{\eta^{2} \beta^{3}}, A_{22}=\frac{(\eta \alpha \beta-k m)^{2} \sigma_{2}^{2}}{\eta^{2} \beta^{4} \gamma_{2}^{2}}
$$

and

$$
\Delta=a^{2}-\frac{4 m}{\eta} b, a=\frac{k m}{\eta \beta}, b=\frac{\eta \alpha \beta-k m}{\beta}, \lambda_{1}=\frac{-a+\sqrt{\Delta}}{2}, \lambda_{2}=\frac{-a-\sqrt{\Delta}}{2} .
$$

Therefore, $D(t)$ converges exponentially to the limiting variance:

$$
D(\infty)=\left[\begin{array}{cc}
\frac{1}{2 \eta \beta^{2} k}\left\{\frac{m \beta \sigma_{1}^{2}}{\gamma_{1}^{2}}+\frac{(\eta \alpha \beta-k m) \sigma_{2}^{2}}{\eta \gamma_{2}^{2}}\right\} & \frac{(k m-\eta \alpha \beta) \sigma_{2}^{2}}{2 \eta^{2} \beta^{3} \gamma_{2}^{2}} \\
\frac{(k m-\eta \alpha \beta) \sigma_{2}^{2}}{2 \eta^{2} \beta^{3} \gamma_{2}^{2}} & \frac{(\eta \alpha \beta-k m)}{\beta^{2}}\left\{\frac{\sigma_{1}^{2}}{2 k \gamma_{1}^{2}}+\frac{\left(k^{2} m+\eta \beta(\eta \alpha \beta-k m)\right) \sigma_{2}^{2}}{2 \eta^{2} \beta^{2} k m \gamma_{2}^{2}}+\frac{4 \sigma}{\eta}\right\}
\end{array}\right] .
$$

This convergence is rapid except when $k$ is close to zero. In the case of high-amplitude fluctuations $D_{11}(\infty)$ and $D_{22}(\infty)$ are large. This indicates that for high-amplitude fluctuations the system demonstrates statistical parametric shatter as a result of rapidly fluctuating environmental conditions, and the equilibrium, which is stable in absence of these fluctuations, becomes unstable. This parametric shatter may occur not only for high-amplitude fluctuations, but also for high fertility to the prey and for small $\eta, \beta$.

5.1. Special case: Volterra-Lotka system. Now using (19) to (22), we have

$$
\begin{aligned}
& \lim _{k \rightarrow 0} D_{11}(t)=\frac{1}{4} \sqrt{\frac{m}{\alpha}} \frac{1}{\eta^{2} \beta^{2}}\left[8 \sigma \sqrt{m \alpha}\{\cos (2 t \sqrt{m \alpha})-1\}+\left\{\frac{m \sigma_{1}^{2}}{\gamma_{1}^{2}}-\frac{\alpha \sigma_{2}^{2}}{\gamma_{2}^{2}}\right\} \sin (2 t \sqrt{m \alpha})\right] \\
& +\frac{m\left(m \sigma_{1}^{2} \gamma_{2}^{2}+\alpha \sigma_{2}^{2} \gamma_{1}^{2}\right)}{2 \eta^{2} \beta^{2} \gamma_{1}^{2} \gamma_{2}^{2}} t
\end{aligned}
$$

and 


$$
\begin{aligned}
& \lim _{k \rightarrow 0} D_{22}(t)=-\frac{1}{4} \sqrt{\frac{\alpha}{m}} \frac{1}{\beta^{2}}\left[8 \sigma \sqrt{m \alpha}\{\cos (2 t \sqrt{m \alpha})-1\}+\left\{\frac{m \sigma_{1}^{2}}{\gamma_{1}^{2}}-\frac{\alpha \sigma_{2}^{2}}{\gamma_{2}^{2}}\right\} \sin (2 t \sqrt{m \alpha})\right] \\
& +\frac{\alpha\left(m \sigma_{1}^{2} \gamma_{2}^{2}+\alpha \sigma_{2}^{2} \gamma_{1}^{2}\right)}{2 \beta^{2} \gamma_{1}^{2} \gamma_{2}^{2}} t .
\end{aligned}
$$

From the above results, we see that as $k \rightarrow 0+$ the damped Volterra-Lotka system tends to a classical Volterra-Lotka system in a random environment which demonstrates statistical parametric shatter with increasing time with a periodic background noise.

\section{Discussion And Conclusion}

In this paper, we have studied the stability behaviour of the damped Volterra-Lotka prey-predator system where prey population lives in herd in a rapidly fluctuating random environment. The method is based on the technique of perturbation approximation of non-linear coupled stochastic differential equations. The characteristic of group-living of prey population has been emphasized using square root of prey density in the functional response. The assumption of condition (13) implies that the system (in deterministic environment) is locally asymptotically stable at the unique non-trivial equilibrium point given by (6). From (13) it is evident that for predator death rates $m$ less than yet not too far from the rate $\eta \beta$ it consumes prey, the predator and prey coexist in stable equilibrium. This is reasonable since, because $m$ is only moderate in size (in deterministic environment), the predator can sufficiently sustain itself yet not grow too much so as to wipe out the prey. Ultimately though, the coexistence necessarily becomes unstable when the predator death rate gets smaller in deterministic environment. In real environment, the coexistence of populations has immense importance for ecological balance in nature. From this viewpoint, study of the stability of the interior equilibrium is emphasized. The analysis indicates that for high-amplitude fluctuations the system demonstrates statistical parametric shatter as a result of rapidly fluctuating environmental conditions, and the equilibrium, which is stable in the absence of these fluctuations, becomes unstable. It is also evident from (15) that the prey population is more sensitive in rapidly fluctuating environmental conditions and the natural growth coefficient $(\alpha)$ of the prey play a significant role in instability (causing ecological imbalance in nature). This is due to the herd behaviour of the prey population.

In Section 5, it is derived that in absence of herd behaviour, the proposed system tends to a classical Volterra-Lotka system for large carrying capacity (of the prey population) in a rapidly fluctuating random environment which demonstrates statistical parametric shatter with increasing time with a periodic background noise. These results are in good agreement with the results obtained by Baishya and Chakrabarti (1987), Samanta (1996), Samanta and Maiti (2003). It is pointed out that this phenomenon does not occur in presence of herd behaviour among the individuals of prey population. So, we have come to the conclusion that when the individuals of prey population act collectively as part of a group, the system develops an internal mechanism to resist this statistical parametric shatter (causing ecological imbalance in nature). It is of course a new result.

\section{Acknowledgements}

The authors are grateful to the anonymous referees and the Chief Editor Prof. Xingfu Zou for their careful reading, valuable comments and helpful suggestions, which have helped them to improve the presentation of this work significantly. 


\section{REFERENCES}

[1] V. Ajraldi, M. Pittavino, E. Venturino, Modelling herd behavior in population systems, Nonlinear Analysis RWA 12(2011), 2319-2338.

[2] M. C. Baishya, C. G. Chakrabarti, Non-equilibrium fluctuation in Volterra-Lotka systems, Bull. Math. Biol. 49(1987), 125-131.

[3] S. P. Bera, A. Maiti, G. P. Samanta, Modelling herd behavior of prey: analysis of a prey-predator model, World Journal of Modelling and Simulation 11(2015), 3-14.

[4] S. P. Bera, A. Maiti, G. P. Samanta Stochastic analysis of a prey-predator model with herd behaviour of prey, Nonlinear Analysis: Modelling and Control 21(2016), 345-361.

[5] S. P. Bera, A. Maiti, G. P. Samanta, Dynamics of a food chain model with herd behaviour of the prey, Model. Earth Syst. Environ. 2(2016):131.

[6] A. P. Braza, Predator-prey dynamics with square root functional responses, Nonlinear Analysis RWA 13(2012),18371843.

[7] C. Cosner C, D. L. DeAngelis, J. S. Ault, D. B. Olson Effects of Spatial Grouping on the Functional Response of Predators, Theor. Pop. Biol. 56(1999), 65-75.

[8] D. L. DeAngelis, W. M. Mooij, Individual-Based Modeling of Ecological and Evolutionary Processes, Annual Review of Ecology, Evolution and Systematics 36(2005),147-168.

[9] M. F. Dimentberg, Statistical Dynamics of Non-linear and Time-Varying Systems, John Wiley and Sons, New York, 1988.

[10] H. I. Freedman, G. Wolkowitz, Predator-prey systems with group defence: the paradox of enrichment revisited, Bull. Math. Biol. 48(1986), 493-508.

[11] J. M. Fryxell, A. Mosser, A. R. E. Sinclair, C. Packer, Group formation stabilizes predator-prey dynamics, Nature 449(2007), 1041-1043.

[12] Q. J. A. Khan, E. Balakrishnan, G. C. Wake Analysis of a predator-prey system with predator switching, Bull. Math. Biol. 66(2004),109-123.

[13] A. Maiti, P. Sen, G. P. Samanta, Deterministic and stochastic analysis of a preypredator model with herd behaviour in both, Systems Science and Control Engineering 4(2016), 259-269.

[14] G. P. Samanta, Influence of environmental noises on the Gomatam model of interacting species, Ecological Modelling 91(1996), 283-291.

[15] G. P. Samanta, M. Maiti, Stochastic gomatam model of interacting species: Non-equilibrium fluctuation and stability, Systems Analysis Modelling Simulation 43(2003), 683-692.

[16] B. S. White, The effects of a rapidly fluctuating random environment on systems of interacting species, SIAM J. Appl. Math. 32(1977), 666-693.

Corresponding author. Department of Mathematics, Indian Institute of Engineering Science and TechNOLOGY, SHIBPUR, HOWRAH - 711103, INDIA

E-mail address: g-p_samanta@yahoo.co.uk, gpsamanta@math.iiests.ac.in

Department of Mathematics, Indian Institute of Engineering Science and Technology, Shibpur, Howrah 711103, INDIA

E-mail address: ashoke.2012@yahoo.com

Department of Mathematics, Indian Institute of Engineering Science and Technology, Shibpur, Howrah 711103, INDIA

E-mail address: debgopalsahoo94@gmail.com

Department of Mathematics, Indian Institute of Engineering Science and Technology, Shibpur, Howrah 711103, INDIA

E-mail address: pralay_dolai@yahoo.in 